\title{
Position Accuracy Analysis on Disc Storing Mechanism for Benthic Drill
}

\author{
Y. Guo ${ }^{*}, 1,2$, Y.P. Jin ${ }^{1}$, M. Jiang ${ }^{1}$ and B.W. Luo ${ }^{1}$ \\ ${ }^{1}$ Hunan Provincial Key Laboratory of Health Maintenance for Mechanical Equipment, Hunan University of Science and \\ Technology, HuNan Xiangtan 411201, China \\ ${ }^{2}$ Key Laboratory of Lightweight and Reliability Technology for Engineering Vehicle, Education Department of Hunan \\ Province, Changsha University of Science \& Technology, HuNan Changsha 410004, China
}

\begin{abstract}
The positional accuracy of disc storing mechanism for benthic drill is the guarantee of long hole coring in deep sea. Aiming the lack of positional accuracy analysis on disc storing mechanism, the mathematic model of the positional accuracy for disc storing mechanism is presented by using complex vector and matrix analyzing method. The analytical formula of crank rotation positional accuracy is acquired through rotation positional analysis of crank in disc storing mechanism driven by hydraulic cylinder. Adopting numerical nonlinear iteration solution method of Newton-Simpson, the variation rule of rotation positional error for disc storing mechanism to cylinder length is acquired, which supports an important theory, leading to tolerance design for dimensional parameters of disc storing mechanism.
\end{abstract}

Keywords: Benthic drill, complex vector, disc storing mechanism, Newton-Simpson, positional error.

\section{INTRODUCTION}

Benthic drill coring technology is currently the most potential marine resources exploration technology. With the increase of marine resources exploration depth, benthic coring drill rig has become the basic equipment in scientific investigation of deep sea, which supplies a strong basis for marine resources exploration [1-5]. The disc storing mechanism is the key part to guarantee the deep sea coring fulfillment in single sampling depth. The positional accuracy of the mechanism directly affects the coring pipe collection and exploration task completion. Therefore, in order to ensure the working reliability of disc storing mechanism, the positional accuracy analysis of the disc storing mechanism is absolutely necessary, which supply a great value to efficient completion of multiple core sampling, and a guarantee to deeper sea exploration. The disc storing mechanism is composed by swing cylinder, disc handle device, coring pipe, connecting base, transmission cylinder, and positional cylinder. Studies of similar mechanisms are mainly focused on kinematics simulation and motion control. Zhang [6] adopted virtue prototype technology to simulate the drill pipe transforms. Using a computer to simulate the actual motion of the drill pipe conveying system, Sha [7] analyzed the motion relation of each component. The displacement, velocity and acceleration of each component are also acquired providing a basis for the optimization of the mechanism. Molfno [8] designed the monitoring system of drill rods delivery. Liu [9] studied the kinematics of each working state of rod storing mechanism, and introduced the automation control system of the mechanism. However, the

*Address correspondence to this author at the Hunan Provincial Key Laboratory of Health Maintenance for Mechanical Equipment, Hunan university of science and technology, HuNan Xiangtan 411201, China;

Tel: +86-15974184466; Fax: +86-0731-58290624;

E-mail: hnkjdx_guoy@163.com positional accuracy analysis of disc storing mechanism driven by hydraulic cylinder has not been studied yet.

The present study is organized as follows. First, the structure and working principle of disc storing mechanism is described. Then, the mathematic model of the positional accuracy for disc storing mechanism is presented by using complex vector and matrix analyzing method. Finally, in order to get the variation rule of rotation positional error for disc storing mechanism to cylinder length, the simulation using the model is done. These supply a lead to tolerance design for dimensional parameters of disc storing mechanism.

\section{STRUCTURE AND WORKING PRINCIPLE FOR DISC STORING MECHANISM}

The disc storing mechanism is shown in Fig. (1). It is composed of swing cylinder, disc handle device, coring pipe, connecting base, transmission cylinder, and positional cylinder. Many coring pipes are stored in disc storing mechanism to implement single deep hole sampling. Swing cylinder and positional cylinder are interlocked with each other. When the mechanism works in the status of transposition, transmission cylinder extends and the positional cylinder retracts. The chamber of swing cylinder without piston rod connects with high pressure. The piston rod of swing cylinder drives disc handle device turning, and transmission cylinder rotates a fixed axis. The transposition completes with the piston rod of swing cylinder as fully extended.

When the mechanism works in the status of return, transmission cylinder retracts and the positional cylinder extends. The chamber of swing cylinder with piston rod connects at high pressure. The piston rod of swing cylinder drives disc handle device turning, and transmission cylinder 
rotates a fixed axis. The return completes with the piston rod of swing cylinder as fully retracted.

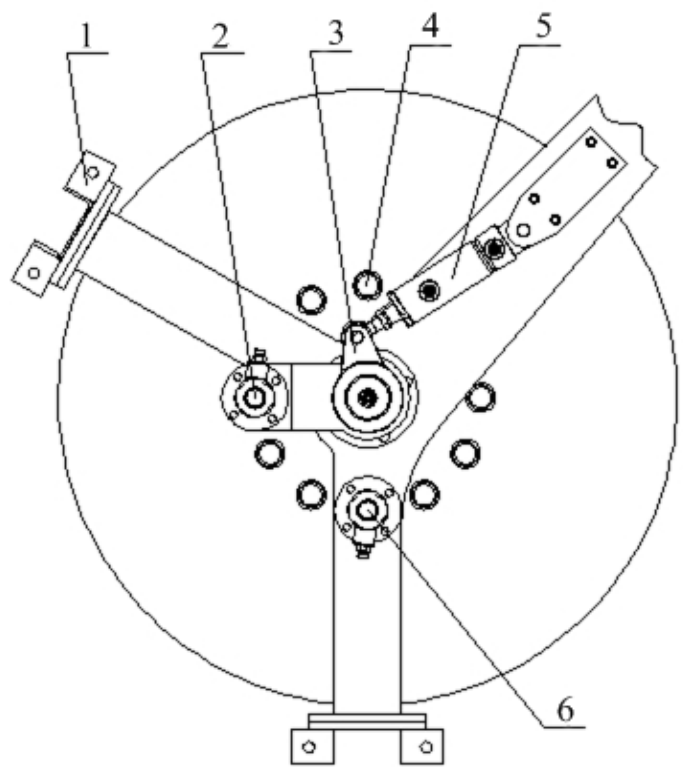

1. connecting base 2 . transmission cylinder 3 . disc handle device

4. coring pipe 5 . swing cylinder 6 . positional cylinder

Fig. (1). Structure of disc storing mechanism.

The disc storing mechanism is driven by swing cylinder. Neglecting the effect of piston rod length, the piston rod is treated as a lumped mass. Consequently, the piston rod can be equivalent to a slider, and the engaging lugs at the ends of rod can be equivalent to revolve pair. The sketch of movement for disc storing mechanism is shown in Fig. (2). In the mechanism, slider is the driver, and rod $\mathrm{AO}$ is the follower. The mechanism makes the linear motion of the swing cylinder to turn the rotation of rod $\mathrm{AO}$, which will realize the coring pipe transposition. $L_{A B O}$ is the initial length of rod $\mathrm{AB}$, and is equal to the length of swing cylinder barrel.

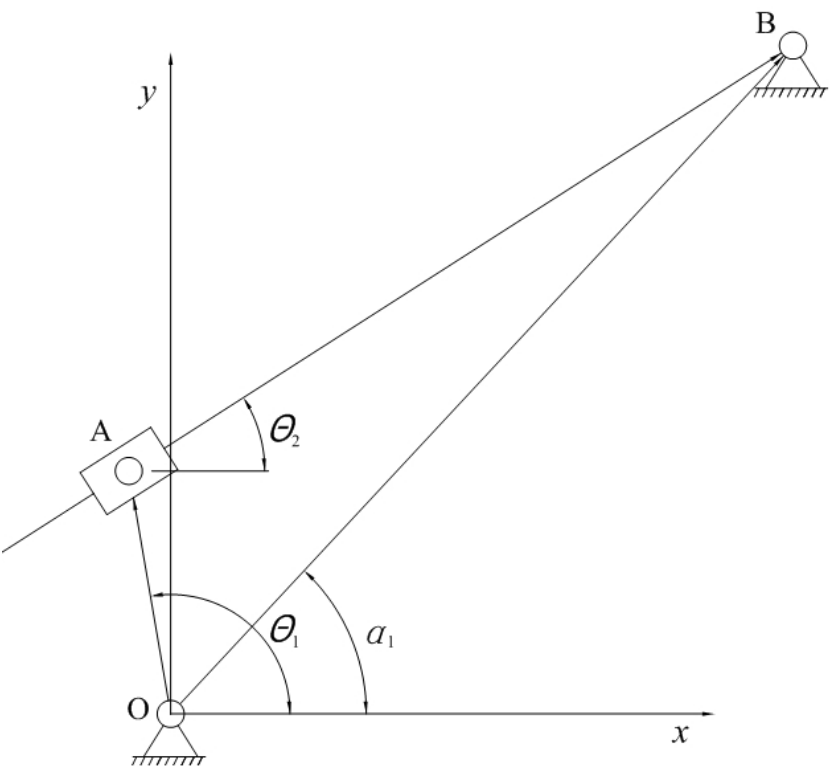

\section{CALCULATION MODEL FOR POSITIONAL ACCURACY ANALYZING DISC STORING MECHANISM}

\subsection{Analytical Theory for Positional Accuracy Analyzing of Disc Storing Mechanism}

In disc storing mechanism, rod $\mathrm{AO}$ is the follower. The position is changed when the length of the cylinder is changed. The position formulation for disc storing mechanism can be written as follows:

$\mathbf{f}(\mathbf{q}, \mathbf{l}, \mathbf{x})=0$

where, $\mathbf{f}=\left(f_{1}, f_{2}, \cdots, f_{n}\right)^{T}$ is the positional function composed by functions with numerical value $n . \mathbf{l}=\left(l_{1}, l_{2}, \cdots, l_{m}\right)^{T}$ is $m$ dimensional vector for the relative poison of dimensional parameters and kinetic pairs. $\mathbf{q}=\left(q_{1}, q_{2}, \cdots, q_{c}\right)^{T}$ is $c$ dimensional imputing vector of the mechanism with $c$ freedom. $\mathbf{x}=\left(x_{1}, x_{2}, \cdots, x_{n}\right)^{T}$ is $n$ dimensional vector for position of the follower.

Using " ${ }^{\circ}$ to denote parameters of ideal mechanism, the $m$ dimensional vector for the relative position of dimensional parameters and kinetic pairs in ideal mechanism is expressed as $\mathbf{I}^{0}, \mathbf{l}^{0}=\left(l_{1}^{0}, l_{2}^{0}, \cdots, l_{m}^{0}\right)^{T}$. The $c$ dimensional imputing vector of the mechanism with $c$ freedom in ideal mechanism is expressed as $\mathbf{q}^{0}, \mathbf{q}^{0}=\left(q_{1}^{0}, q_{2}^{0}, \cdots, q_{c}^{0}\right)^{T}$, and the $n$ dimensional vector for position of the follower in ideal vector is expressed as $\mathbf{x}^{0}, \mathbf{x}^{0}=\left(x_{1}^{0}, x_{2}^{0}, \cdots, x_{n}^{0}\right)^{T}$. Accordingly, the dimensional error can be expressed as follows. Accordingly, the dimensional error of components can be expressed as follows:

$\Delta \mathbf{l}=\left(\Delta l_{1}, \Delta l_{2}, \cdots \Delta l_{m}\right)^{T}=\left(l_{1}-l_{1}^{0}, l_{2}-l_{2}^{0}, \cdots l_{m}-l_{m}^{0}\right)^{T}$

The positional error of driver to ideal mechanism can be expressed as follows:

$\Delta \mathbf{q}=\left(\Delta q_{1}, \Delta q_{2}, \cdots \Delta q_{c}\right)^{T}=\left(q_{1}-q_{1}^{0}, q_{2}-l_{2}^{0}, \cdots q_{c}-q_{c}^{0}\right)^{T}$

Therefore, the position error of the follower can be written as:

$\Delta \mathbf{x}=\left(\Delta x_{1}, \Delta x_{2}, \cdots \Delta x_{n}\right)^{T}=\left(x_{1}-x_{1}^{0}, x_{2}-x_{2}^{0}, \cdots x_{n}-x_{n}^{0}\right)^{T}$

The actual mechanism and ideal mechanism should meet the same position equations. Hence, we can get:

$\mathbf{f}\left(\mathbf{q}^{0}, \mathbf{l}^{0}, \mathbf{x}^{0}\right)=0$

where, $\mathbf{f}^{0}=\left(f_{1}^{0}, f_{2}^{0}, \cdots f_{3}^{0}\right)^{T}$ is the vector of positional function composed by functions with value $n$. According to Eq. (1) and Eq. (5), we can get:

$$
\begin{aligned}
& \Delta \mathbf{f}\left(\mathbf{q}-\mathbf{q}^{0}, \mathbf{l}-\mathbf{l}^{0}, \mathbf{x}-\mathbf{x}^{0}\right)=0 \\
& \mathbf{f}\left(\mathbf{q}^{0}+\Delta \mathbf{q}, \mathbf{l}^{0}+\Delta \mathbf{l}, \mathbf{x}^{0}+\Delta \mathbf{x}\right)-\mathbf{f}\left(\mathbf{q}^{0}, \mathbf{l}^{0}, \mathbf{x}^{0}\right)=0
\end{aligned}
$$

It also can be written as:

Fig. (2). Sketch of disc storing mechanism. 
$f\left(q_{1}^{0}+\Delta q_{1}, \cdots, q_{c}^{0}+\Delta q_{c}, l_{1}^{0}+\Delta l_{1}, \cdots, l_{m}^{0}+\Delta l_{m}, x_{1}^{0}+\Delta x_{1}, \cdots, x_{n}^{0}+\Delta x_{n}\right)=0(8)$

The left part of Eq. (8) is nonlinear functions. The errors of $\Delta \mathbf{q}, \Delta \mathbf{l}$ and $\Delta \mathbf{x}$ are very small. Expanding Eq. (8) on $\mathbf{q}^{0}$

, $\mathbf{l}^{0}$ and $\mathbf{x}^{0}$, the equation is changed to follow by neglecting high-order trace.

$\sum_{k=1}^{c} \frac{\partial f_{i}^{0}}{\partial q_{k}^{0}} \Delta q_{k}+\sum_{s=1}^{m} \frac{\partial f_{i}^{0}}{\partial l_{s}^{0}} \Delta l_{s}+\sum_{j=1}^{n} \frac{\partial f_{i}^{0}}{\partial x_{j}^{0}} \Delta x_{j}=0 \quad(i=1,2, \cdots, n)$

where, $\frac{\partial f^{0}}{\partial q^{0}}, \frac{\partial f^{0}}{\partial l^{0}}$ and $\frac{\partial f^{0}}{\partial x^{0}}$ are values of the partial derivatives on $\left(\mathbf{q}^{0}, \mathbf{l}^{0}, \mathbf{x}^{0}\right)$, which are not relative to errors of $(\Delta \mathbf{q}, \Delta \mathbf{l}, \Delta \mathbf{x})$.

Obviously, the positional error of the follower of disc storing mechanism is a linear combination of the original error. Each item in formulate is the position error of each one corresponding to the original error. The Eq. (9) can be written as follows using matrix.

$\mathbf{D}_{\mathbf{q}}^{0} \Delta \mathbf{q}+\mathbf{D}_{\mathbf{1}}^{0} \Delta \mathbf{l}+\mathbf{D}_{\mathbf{x}}^{0} \Delta \mathbf{x}=0 \quad(i=1,2, \cdots, n)$ as:

Hence, the positional error of the follower can be written

$$
\Delta \mathbf{x}=-\mathbf{D}_{\mathbf{x}}^{\mathbf{0}-1} \mathbf{D}_{\mathbf{q}}^{\mathbf{0}} \Delta \mathbf{q}-\mathbf{D}_{\mathbf{x}}^{0-1} \mathbf{D}_{1}^{0} \Delta \mathbf{l}
$$

where, $\mathbf{D}_{\mathbf{x}}=\left(\frac{\partial \mathbf{f}_{\mathbf{i}}}{\partial \mathbf{x}_{\mathbf{j}}}\right)_{n \times n}$ is the $n \times n$ dimensional partial matrix of $\mathbf{f}(\mathbf{q}, \mathbf{l}, \mathbf{x})$ to $\mathbf{x} . \quad \mathbf{D}_{q}=\left(\frac{\partial \mathbf{f}_{\mathbf{i}}}{\partial q_{k}}\right)_{n \times c}$ is the $n \times c$ dimensional partial matrix of $\mathbf{f}(\mathbf{q}, \mathbf{l}, \mathbf{x})$ to $\mathbf{q} . \mathbf{D}_{l}=\left(\frac{\partial \mathbf{f}_{\mathbf{i}}}{\partial l_{s}}\right)_{n \times m}$ is the $n \times m$ dimensional partial matrix of $\mathbf{f}(\mathbf{q}, \mathbf{l}, \mathbf{x})$ to $\mathbf{l}$.

The positional error of the follower can be obtained by solving Eq. (11).

\subsection{Positional Accuracy Model of Disc Storing Mechanism}

The coordinate is built as shown in Fig. (2) shown. In the disc storing mechanism, $\alpha_{3}$ is the angle of frame $\mathrm{AB}$ and reference axis $x, l_{1}$ is the length of $\operatorname{rod} \mathrm{OA}, l_{2}$ is the cylinder length, and $l_{3}$ is the distance of point $\mathrm{O}$ and B. $\theta_{i} \quad(i=1,2)$ is the angle of rods to reference axis $x$. According to the vector calculation rule, the positional equation for disc storing mechanism can be described as:

$l_{1} e^{j \theta_{1}}+l_{2} e^{j \theta_{2}}=l_{3} e^{j \alpha_{1}}$

The real and imaginary part of complex is equivalent, respectively, and the positional equation of disc storing mechanism can be equivalent to follow $[10,11]$.

$\left\{\begin{array}{c}f_{1}=l_{1} \cos \theta_{1}+l_{2} \cos \theta_{2}-l_{3} \cos \alpha_{3}=0 \\ f_{2}=l_{1} \sin \theta_{1}+l_{2} \sin \theta_{2}-l_{3} \sin \alpha_{3}=0\end{array}\right.$
In the disc storing mechanism, the cylinder is the driver. The vector of relative position is expressed as $\mathbf{l}$, $\mathbf{I}=\left(l_{1}, l_{3}, \alpha_{1}\right)^{T}$. The imputing vector of the mechanism is expressed as $\mathbf{q}, \mathbf{q}=l_{2}$. The vector for position of the follower is expressed as $\mathbf{x}, \mathbf{x}=\left(\theta_{1}, \theta_{2}\right)^{T}$. Hence, the dimensional error of components can be expressed as $\boldsymbol{\Delta l}, \boldsymbol{\Delta l}=\left(\Delta l_{1}, \Delta l_{3}, \Delta \alpha_{1}\right)^{T}$, the positional error of driver to ideal mechanism can be expressed as $\Delta \mathbf{q}, \Delta \mathbf{q}=\Delta l_{2}$, and the position error of the follower can be written as $\Delta \mathbf{x}, \Delta \mathbf{x}=\left(\Delta \theta_{1}, \Delta \theta_{2}\right)^{T}$. According to Eq. (11), the output error of the disc storing mechanism can be expressed as

$$
\begin{aligned}
{\left[\begin{array}{l}
\Delta \theta_{1} \\
\Delta \theta_{2}
\end{array}\right]=\left[\begin{array}{cc}
-l_{1} \sin \theta_{1} & -l_{2} \sin \theta_{2} \\
l_{1} \cos \theta_{1} & l_{2} \cos \theta_{2}
\end{array}\right]^{-1}\left[\begin{array}{c}
\cos \theta_{1} \\
\sin \theta_{2}
\end{array}\right] \Delta l_{2} } \\
+\left[\begin{array}{cc}
-l_{1} \sin \theta_{1} & -l_{2} \sin \theta_{2} \\
l_{1} \cos \theta_{1} & l_{2} \cos \theta_{2}
\end{array}\right]^{-1}\left[\begin{array}{ccc}
\cos \theta_{1} & \cos \alpha_{1} & l_{3} \sin \alpha_{1} \\
\sin \theta_{1} & -\sin \theta_{1} & -l_{3} \cos \alpha_{1}
\end{array}\right]\left[\begin{array}{c}
\Delta l_{1} \\
\Delta l_{2} \\
\Delta \alpha_{1}
\end{array}\right]
\end{aligned}
$$

\section{POSITIONAL ACCURACY ANALYSIS OF DISC STORING MECHANISM FOR BENTHIC CORING DRILL}

\section{1 .Analyzing Parameters}

According to the length of rods and the tolerance requirements for disc storing mechanism, the analyzing parameters are shown as follows. The length of disc handle device is $l_{1}=148 \pm 1 \mathrm{~mm}$. The initial length of cylinder is $l_{20}=474 \pm 2 \mathrm{~mm}$. The distance of joint $\mathrm{O}$ and $\mathrm{B}$ is $l_{3}=474 \pm 2 \mathrm{~mm}$. The angle of frame AB and reference axis $x$ is $\alpha_{1}=47 \pm 0.5^{\circ}$. In initial condition, the evaluated angle of disc handle device and reference axis $x$ is $\theta_{1}=100^{\circ}$, and the evaluated angle of cylinder and reference axis $x$ is $\theta_{2}=33^{\circ}$.

\subsection{Numerical Solution Method}

According to Eq. (13), the solution of $\theta_{1}$ and $\theta_{2}$ is obtained using Neton-Simpson solving method. The variable

$\theta_{i}$ is described as the sum of estimates of solutions $\bar{\theta}_{i}$ and tiny correction factor $\Delta \theta_{i}$ for the difference between prediction values and estimate values.

Expand Eq. (13) using Taylor series, the Eq. (13) is changed to

$$
\left[\begin{array}{c}
f_{1}\left(\bar{\theta}_{1}, \bar{\theta}_{2}\right) \\
f_{2}\left(\bar{\theta}_{1}, \bar{\theta}_{2}\right)
\end{array}\right]+\left[\begin{array}{cc}
\left.\frac{\partial f_{1}}{\partial \theta_{1}}\right|_{\theta_{1}, \bar{\theta}_{2}} & \left.\frac{\partial f_{1}}{\partial \theta_{2}}\right|_{\theta_{1}, \bar{\theta}_{2}} \\
\left.\frac{\partial f_{2}}{\partial \theta_{1}}\right|_{-\overline{\theta_{1},}, \theta_{2}} & \left.\frac{\partial f_{2}}{\partial \theta_{2}}\right|_{\bar{\theta}_{1}, \bar{\theta}_{2}}
\end{array}\right]\left[\begin{array}{c}
\Delta \theta_{1} \\
\Delta \theta_{2}
\end{array}\right]=\left[\begin{array}{l}
0 \\
0
\end{array}\right]
$$


Therefore, tiny correction factor $\Delta \theta_{i}$ for the difference between prediction values and estimate values can be expressed as:

$\left[\begin{array}{c}\Delta \theta_{1} \\ \Delta \theta_{2}\end{array}\right]=-\left[\begin{array}{cc}\left.\frac{\partial f_{1}}{\partial \theta_{1}}\right|_{\bar{\theta}_{1}, \overline{\theta_{2}}} & \left.\frac{\partial f_{1}}{\partial \theta_{2}}\right|_{\bar{\theta}_{1}, \overline{\theta_{2}}} \\ \left.\frac{\partial f_{2}}{\partial \theta_{1}}\right|_{\bar{\theta}_{1}, \overline{\theta_{2}}} & \left.\frac{\partial f_{2}}{\partial \theta_{2}}\right|_{\bar{\theta}_{1}, \bar{\theta}_{2}}\end{array}\right]^{-1}\left[\begin{array}{c}f_{1}\left(\overline{\theta_{1}}, \overline{\theta_{2}}\right) \\ f_{2}\left(\bar{\theta}_{1}, \overline{\theta_{2}}\right)\end{array}\right]$

Reset the estimates of solutions $\bar{\theta}_{i}$ and iterate repeatedly. When the tiny correction factor $\Delta \theta_{i}$ for the difference between prediction values and estimate values, reaches to the set accuracy, the solution completes and the estimates of solutions $\bar{\theta}_{i}$ are the equivalent solution $\theta_{i}$ of the equation $[12,13]$.

Solving Eq. (14), gives the positional error of disc storing mechanism. The whole numerical solving scheme is shown as Fig. (3).

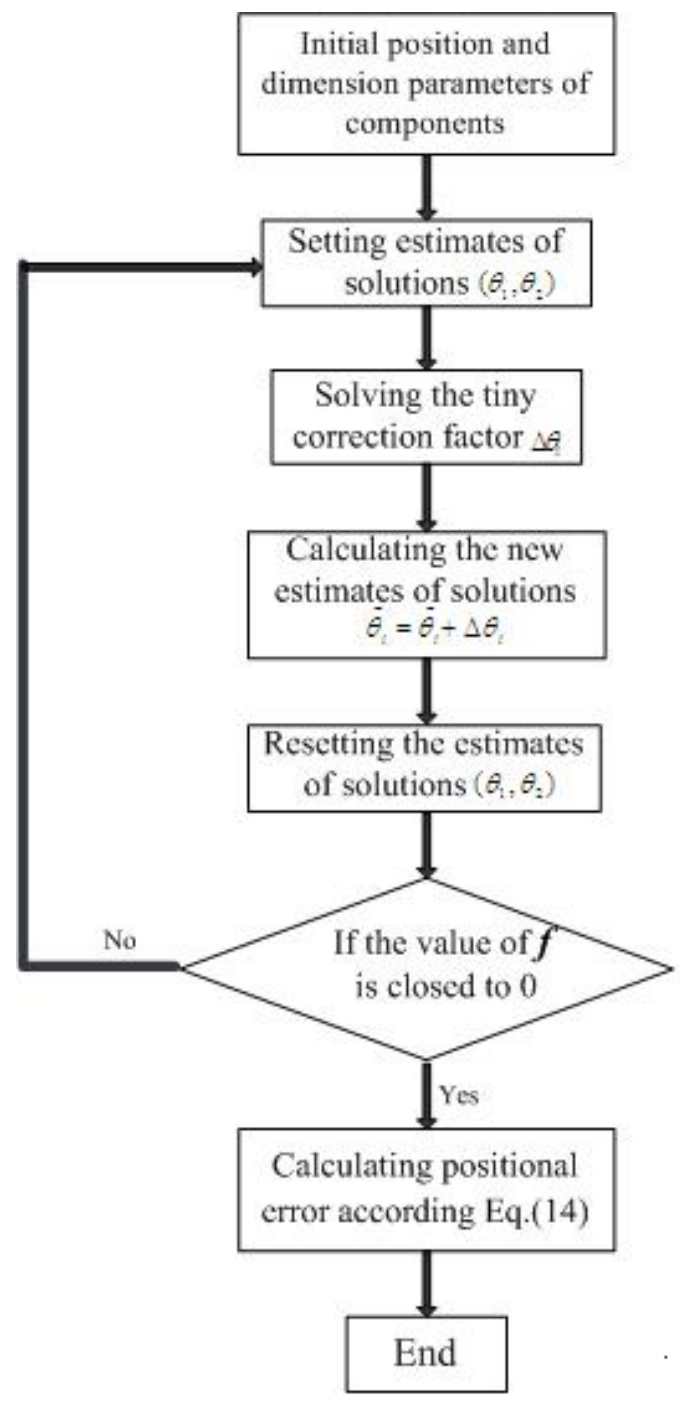

Fig. (3). Whole numerical solving scheme.

\subsection{Calculation Results of Positional Error}

In working process, the length of cylinder $l_{2}$ for disc storing mechanism is changed from $474 \mathrm{~mm}$ to $550 \mathrm{~mm}$. Increasing the cylinder length $\Delta l_{2}$ and setting at $5.43 \mathrm{~mm}$, the positional error on each position is shown in Table $\mathbf{1}$, and the curve of positional error to cylinder length changes is shown as Fig. (4). In Table 1, $l_{2}=l_{20}+i \Delta l_{2}$.

Table 1. Positional error of disc storing mechanism.

\begin{tabular}{|c|c|c|c|c|}
\hline $\mathrm{i}$ & $\boldsymbol{\theta}_{\mathbf{1}}{ }^{\circ}$ & $\boldsymbol{\theta}_{\mathbf{2}}{ }^{\circ}$ & $\Delta \boldsymbol{\theta}_{\mathbf{1}}{ }^{\circ}$ & $\Delta \boldsymbol{\theta}_{\mathbf{2}}{ }^{\circ}$ \\
\hline \hline 0 & 99.7948 & 32.6001 & 0.8127 & -0.6805 \\
\hline 1 & 102.0550 & 32.3424 & 0.7507 & -0.6293 \\
\hline 2 & 104.2792 & 32.1194 & 0.6827 & -0.5752 \\
\hline 3 & 106.4735 & 31.9286 & 0.6180 & -0.5235 \\
\hline 4 & 108.6429 & 31.7679 & 0.5617 & -0.4773 \\
\hline 5 & 110.7920 & 31.6354 & 0.5163 & -0.4377 \\
\hline 6 & 112.9249 & 31.5296 & 0.4825 & -0.4051 \\
\hline 7 & 115.0453 & 31.4491 & 0.4604 & -0.3790 \\
\hline 8 & 117.1566 & 31.3928 & 0.4494 & -0.3589 \\
\hline 9 & 119.2621 & 31.3597 & 0.4488 & -0.3441 \\
\hline 10 & 121.3647 & 31.3490 & 0.4582 & -0.3339 \\
\hline 11 & 123.4673 & 31.3598 & 0.4773 & -0.3277 \\
\hline 12 & 125.5727 & 31.3917 & 0.5058 & -0.3250 \\
\hline 13 & 127.6837 & 31.4440 & 0.5438 & -0.3254 \\
\hline 14 & 129.8030 & 31.5165 & 0.5914 & -0.3284 \\
\hline
\end{tabular}

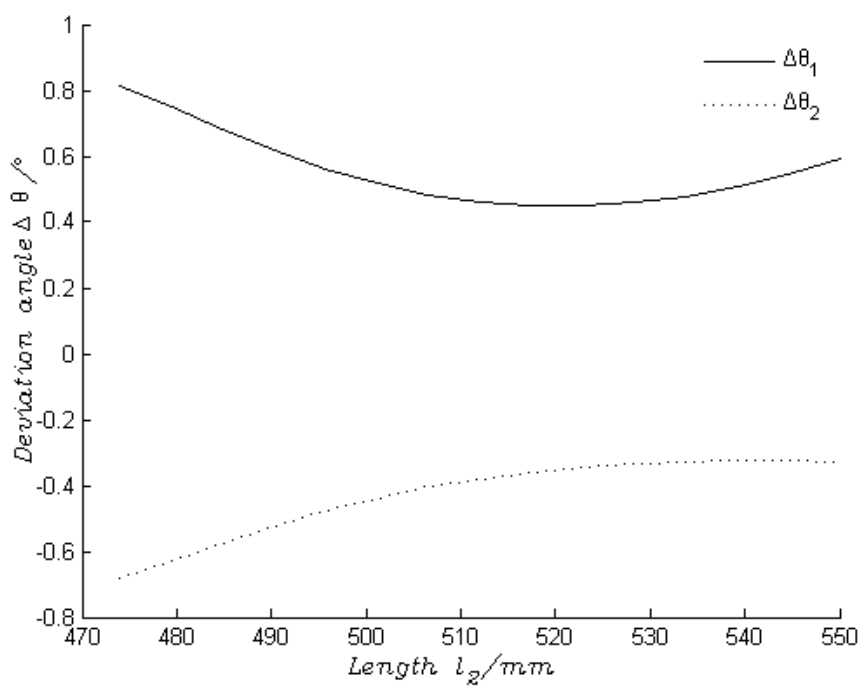

Fig. (4). Positional error to cylinder length changes.

The results show that, the crank rotating angle errors caused by length parameters are positive, which ranges from $0.4488 \mathrm{~mm}$ to $0.8127 \mathrm{~mm}$. The errors of angle for cylinder and reference axis $x$ are negative in the working process, which ranges from $-0.6805 \mathrm{~mm}$ to $-0.3250 \mathrm{~mm}$. The 
maximum crank rotating angle error appears on initial position, $l_{2}=474 \mathrm{~mm}$, and $\theta_{1}=99.7948^{\circ}, \theta_{2}=32.6001^{\circ}$. There is an inflection point on curve for crank rotating angle error to cylinder length. When cylinder length $l_{2}$ is less then $517.44 \mathrm{~mm}$, the crank rotating angle errors decrease with the increase of the cylinder length. However, the crank rotating angle errors increase with the increase of the cylinder length, when cylinder length $l_{2}$ is larger then $517.44 \mathrm{~mm}$. The maximum angle error $\Delta \theta_{2}$ of cylinder to reference axis $x$ is also appears on initial position, $l_{2}=474 \mathrm{~mm}$, and the angle error of cylinder to reference axis $x$ decreases with the increase of the cylinder length.

\section{CONCLUSION}

The mathematical model of positional accuracy for disc storing mechanism is established using complex vector and matrix analyzing method. The crank rotating angle positional accuracy is analyzed using the model, which supports an important theory, leading to tolerance design for dimensional parameters of disc storing mechanism. The results acquired through the analysis are shown as follows:

1) The crank rotating angle errors caused by length parameters are positive, however, the errors of angle for cylinder and reference axis $x$ are negative in the working process.

2) The maximum positional error for angle of crank rotating angle and cylinder to reference axis $x$ both appear at initial position, $l_{2}=474 \mathrm{~mm}$.

3) When cylinder length $l_{2}$ is less then $517.44 \mathrm{~mm}$, the crank rotating angle errors decrease with the increase of the cylinder length. However, the crank rotating angle errors increase with the increase of the cylinder length, when cylinder length $l_{2}$ is larger then 517.44 $\mathrm{mm}$.

4) The angle error of cylinder to reference axis $x$ decreases with the increase of the cylinder length.

\section{CONFLICT OF INTEREST}

The authors confirm that this article content has no conflict of interest.

\section{ACKNOWLEDGEMENTS}

The authors thank the reviewers for their valuable comments and the support by grants from National High Technology Research and Development Program of China (No. 2012AA091301), Key Laboratory of Lightweight and Reliability Technology for Engineering Vehicle, College of Hunan Province (No. 2012KFJJ04), Scientific Research Fund of Hunan Provincial Education Department (No.13C316), Hunan Provincial Key Laboratory of Health Maintenance for Mechanical Equipment (No. 201305), Startup Foundation of High-talent Research Project from HuNan Univercity of Science and Technology (No. E53101), and Hunan Provincial Natural Science Foundation of China (No. 14JJ6029).

\section{REFERENCES}

[1] X.K. Zhu, X.L. Jin, C.H. Tao, F.Y. Chu, and Y.P. Li, "Discussion on development of ocean exploration technologies and equipments", Robot, vol. 35, pp. 376-384, May 2013.

[2] M. Selvakumar, R.R. Subramanian, A.N. Sathianarayanan, G. Jayakumar, D. Harikrishnan, V.K. Muthukumaran, D. Murugesan, M. Chandresekaran, E. Elangovan, D. Prakash, V. Vadivelan, A. Radhakrishnan, M. Ramesh, S. Ramadass, and G.A. Atmanand, "Technology tool for deep ocean exploration-remotely operated vehicle", in Proceedings of the $20^{\text {th }}$ international offshore and polar engineering conference, 2010, pp. 206-212.

[3] D. Yamazaki, D. Alsdorf, K. Hyungjun, S. Kanae, T. Oki, and K. Andreadis, "Toward global-scale data assimilation using swot: requirements for global hydrodynamics models", In Geoscience and remote sensing symposium, Vancouver, 2011, pp. 3031-3034.

[4] L. Zhang, D. P. Jiang, J. X. Zhao, and S. Ma, "An AUV for ocean exploring and its motion control system architecture", The open Mechanical Engineering Journal, Vol. 7, pp. 40-47, Sept, 2013.

[5] K.D. Xu, "Development of ocean engineering technique and exploitation and utilization of marine resources", Ocean Development and Management, vol. 5, pp. 4-7, May, 2002.

[6] Z. X. Zhang, J. H. Liu and R. X. Ning, "Kinematics analysis of mechanisms based on virtual assembly", Chinese Journal of Mechanical Engineering, vol. 23, pp. 748-757, Oct 2010.

[7] Y. B. Sha, J. L. Zhu, Q. Y. Wang and X. Q. Zhao, "Kinematic analysis and simulation research on pipe automatic transferring system", Journal of Mechanical Engineering, vol. 48, pp. 45-49, Sept 2012.

[8] R. M. Molfno, R. P. Razzoli and M. Zoppi, “Autonomous drilling robot for landslide monitoring and consolidation", Automation in Construction, vol. 17, pp. 111-121, Jan 2008.

[9] Q. P. Liu, X. Z. Cui and L. Dong, "Treatment methods and automatic handling systems of drill pipe for drilling platforms", China Offshore Platform, vol. 25, pp. 51-57, Feb 2010.

[10] J. Awrejcewicz, "Classical mechanics: Kinematics and Statics". New York: Springer, 2012.

[11] J. Awrejcewicz, "Classical Mechanics: Dynamics", New York: Springer, 2012.

[12] Q. S. Yan and R. Zheng, "Prediction simulation for the length of garment marking based on QPSO-DFNN", The Open Mechanical Engineeting Journal, Vol.8, pp.31-36, Feb 2014.

[13] N. Khaled, "Virtual Reality and Animation for MATLAB and Simulink Users: Visualization of Dynamic Models and Control Simulations", London, New York: Springer, 2012. 